Homology, Homotopy and Applications, vol.21(1), 2019, pp.1-18

\title{
COALGEBRAS IN SYMMETRIC MONOIDAL CATEGORIES OF SPECTRA
}

\author{
MAXIMILIEN PÉROUX AND BROOKE SHIPLEY
}

(communicated by J.P.C. Greenlees)

\begin{abstract}
We show that all coalgebras over the sphere spectrum are cocommutative in the category of symmetric spectra, orthogonal spectra, $\Gamma$-spaces, $\mathcal{W}$-spaces and EKMM $\mathbb{S}$-modules. Our result only applies to these strict monoidal categories of spectra and does not apply to the $\infty$-category setting.
\end{abstract}

\section{Introduction}

It is well known that the diagonal map of a set, or a space, gives it the structure of a comonoid. In fact, the only possible (counital) comonoidal structure on an object in a Cartesian symmetric monoidal category is given by the diagonal (see [AM10, Example 1.19]). Thus all comonoids are forced to be cocommutative in these settings. We prove that this rigidity is inherited by all of the strict monoidal categories of spectra that have been developed over the last 20 years, including symmetric spectra (see [HSS00]), orthogonal spectra (see [MMSS01, MM02]), Г-spaces (see [Seg74, BF78]), W-spaces (see [And74]) and $\mathbb{S}$-modules (see [EKMM97]), which we call EKMM-spectra here. That is, $\mathbb{S}$-coalgebra spectra in any of these categories are cocommutative, where $\mathbb{S}$ denotes the sphere spectrum.

Theorem 1.1. Let $(C, \Delta, \varepsilon)$ be an $\mathbb{S}$-coalgebra in symmetric spectra, orthogonal spectra, $\Gamma$-spaces, $\mathcal{W}$-spaces or EKMM-spectra. Then $C$ is a cocommutative $\mathbb{S}$-coalgebra.

Furthermore, we prove that all $R$-coalgebras are cocommutative whenever $R$ is a commutative $\mathbb{S}$-algebra with $R_{0}$ homeomorphic to $S^{0}$; see Theorems 3.4 and 4.1 . Moreover, in Remark 3.5, we recall that, in symmetric spectra or orthogonal spectra, every commutative $\mathbb{S}$-algebra is weakly equivalent to one whose zeroth space is homeomorphic to $S^{0}$.

It is important to note that these results are restricted to the listed strict monoidal categories of spectra. In [Lur18], Lurie considers the category of cocommutative coalgebras in a symmetric monoidal $\infty$-category and establishes an equivalence between the associated dualizable algebra and coalgebra objects in [Lur18, Corollary 3.2.5]. One could similarly consider the category of (not necessarily cocommutative)

The second author was supported by the National Science Foundation, DMS-140648.

Received October 1, 2017, revised February 19, 2018; published on August 15, 2018.

2010 Mathematics Subject Classification: 16T15, 18D10, 55P42, 55P43.

Key words and phrases: homotopy, spectrum, coalgebra, comonoid.

Article available at http://dx.doi.org/10.4310/HHA.2019.v21.n1.a1

Copyright (C) 2018, Maximilien Péroux and Brooke Shipley. Permission to copy for private use granted. 
coalgebras and their dualizable objects. Since there exist compact non-commutative $\mathbb{S}$-algebra spectra, one can consider for such an $X$ the Spanier-Whitehead dual, $D X=\operatorname{hom}(X, \mathbb{S})$, and this represents a non-cocommutative $\mathbb{S}$-coalgebra spectrum. This shows that the category of coalgebras in the $\infty$-category of spectra has noncocommutative objects.

Given a symmetric monoidal model category $\mathcal{M}$, the underlying $\infty$-category, $\overline{\mathcal{N}}$, admits a symmetric monoidal structure. As part of his $\mathrm{PhD}$ thesis, the first author is determining conditions that imply the equivalence of the $\infty$-category of (cocommutative) coalgebras over $\overline{\mathcal{M}}$ and the nerve of (cocommutative) coalgebras in $\mathcal{M}$. In other words, this compares the coalgebra objects with a (cocommutative) comultiplication up to coherent homotopy with the strictly (cocommutative) coalgebras.

The paper is organized as follows. In Section 2, we recall the notions of comonoids and coalgebras and introduce a purely categorical argument, Theorem 2.2, which is at the heart of the proof of Theorem 1.1. Section 3 considers the general setting of diagram spectra introduced in [MMSS01] which encapsulates most of the categories of spectra mentioned above. The category of EKMM-spectra needs particular care and is considered in Section 4.

\section{Acknowledgments}

We are grateful for initial conversations on these topics with Kathryn Hess. We also thank Ben Antieau for reading and commenting on early versions of this paper. The authors would like to thank the referee for helpful comments on the preliminary version of this paper.

\section{Definition and preliminaries}

Let $(\mathcal{C}, \otimes, \mathbb{I})$ be a symmetric monoidal category throughout this section.

Definition 2.1. A comonoid $(C, \Delta, \varepsilon)$ in $\mathcal{C}$ consists of an object $C$ in $\mathcal{C}$ together with a coassociative comultiplication $\Delta: C \rightarrow C \otimes C$, such that the following diagram commutes:

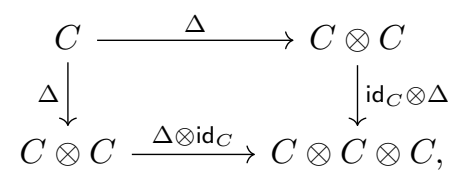

and admits a counit morphism $\varepsilon: C \rightarrow \mathbb{I}$ such that we have the following commutative diagram:

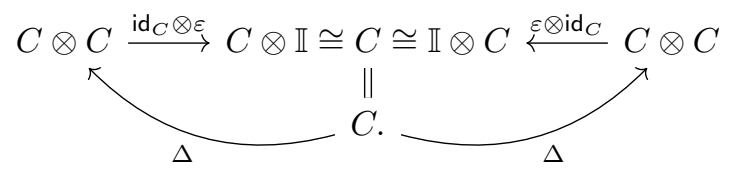

The comonoid is cocommutative if the following diagram commutes:

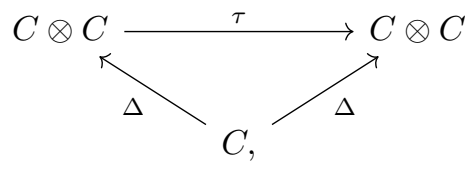


where $\tau$ is the twist isomorphism from the symmetric monoidal structure of $\mathrm{C}$. A morphism of comonoids $f:(C, \Delta, \varepsilon) \rightarrow\left(C^{\prime}, \Delta^{\prime}, \varepsilon^{\prime}\right)$ is a morphism $f: C \rightarrow C^{\prime}$ in $\mathcal{C}$ such that the following diagrams commute:
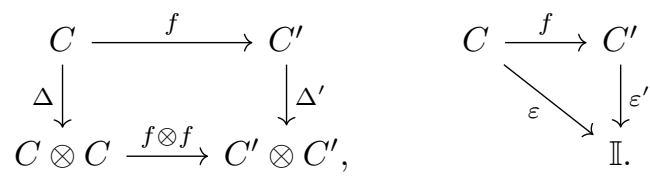

We denote $\operatorname{coMon}(\mathcal{C})$ the category of comonoids in $\mathcal{C}$.

In the next sections, our main strategy uses the following result.

Theorem 2.2. Let $(\mathcal{C}, \otimes, \mathbb{I})$ and $(\mathcal{D}, \odot, \mathbb{J})$ be symmetric monoidal categories endowed with a pair of adjoint underlying functors $L: \mathcal{C} \rightleftarrows \mathcal{D}: R$, where the functor $R$ is lax symmetric monoidal such that:

(i) the maps $\mathbb{I} \rightarrow R(\mathbb{J})$ and $R(D) \otimes R(D) \rightarrow R(D \odot D)$ are isomorphisms in $\mathcal{D}$, for all comonoids $D$ in $\mathcal{D}$;

(ii) all comonoids in $\mathcal{C}$ are cocommutative;

(iii) for each comonoid $D$ in $\mathcal{D}$, the counit map $L R(D) \rightarrow D$ given by the adjunction is an epimorphism in $\mathcal{D}$.

Then all comonoids in $\mathcal{D}$ are cocommutative.

Notice that condition (i) of Theorem 2.2 is respected whenever $R$ is a strong monoidal functor.

Proof. Recall from [AM10, Proposition 3.85], as $R: \mathcal{D} \rightarrow \mathcal{C}$ is a lax symmetric monoidal functor, there exists a unique lax symmetric comonoidal structure on the functor $L: \mathcal{C} \rightarrow \mathcal{D}$ such that the adjoint pair forms a symmetric monoidal conjunction (sometimes called colax-lax adjunction, see [AM10, Definition 3.81]). Since $L$ is lax symmetric comonoidal, it sends (cocommutative) comonoids in $\mathcal{C}$ to (respectively cocommutative) comonoids in $\mathcal{D}$ (see [AM10, Proposition 3.29, Proposition 3.37]). Because of condition (i), one can check that if $(D, \Delta, \varepsilon)$ is a comonoid in $\mathcal{D}$, then $R(D)$ is a comonoid in $\mathcal{C}$ with comultiplication:

$$
R(D) \stackrel{R(\Delta)}{\longrightarrow} R(D \odot D) \stackrel{\cong}{\longleftarrow} R(D) \otimes R(D)
$$

and counit:

$$
R(D) \stackrel{R(\varepsilon)}{\longrightarrow} R(\mathbb{J}) \stackrel{\cong}{\longleftarrow} \mathbb{I} .
$$

Using condition (ii) we get that $L R(D)$ is a cocommutative comonoid in $\mathcal{D}$ for any (not necessarily cocommutative) comonoid $D$ in $\mathcal{D}$. One can show the counit map of the adjoint $L R(D) \rightarrow D$ is a morphism of comonoids in $\mathcal{D}$ as in [AM10, Proposition 3.93]. We conclude using (iii) and Proposition 2.3.

Recall that any subalgebra of a commutative algebra is also commutative. The following is the dual case and could be proved using opposite categories. 
Proposition 2.3. Let $(C, \Delta, \varepsilon)$ and $\left(C^{\prime}, \Delta^{\prime}, \varepsilon^{\prime}\right)$ be comonoids in $(\mathcal{C}, \otimes, \mathbb{I})$. Suppose $C$ is cocommutative. Given a morphism of comonoids $f: C \rightarrow C^{\prime}$, if $f$ is an epimorphism in $\mathcal{C}$, then $C^{\prime}$ is also cocommutative.

Proof. Since $f$ is a morphism of comonoids the top square in the following diagram commutes:

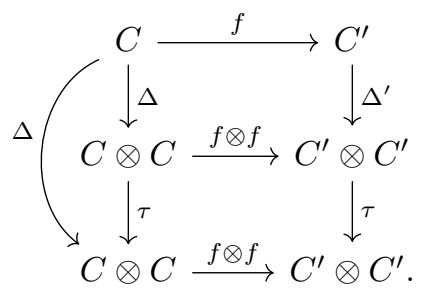

The bottom square commutes from the naturality of the twist isomorphism $\tau$. The left side commutes as $C$ is cocommutative. The commutativity of the above diagram gives:

$$
\tau \circ \Delta^{\prime} \circ f=\tau \circ(f \otimes f) \circ \Delta=(f \otimes f) \circ \tau \circ \Delta=(f \otimes f) \circ \Delta=\Delta^{\prime} \circ f .
$$

Since $f$ is an epimorphism in $\mathcal{C}$, it follows that $\Delta^{\prime}=\tau \circ \Delta^{\prime}$. Therefore $\left(C^{\prime}, \Delta^{\prime}, \varepsilon^{\prime}\right)$ is cocommutative.

Subsequently condition (ii) of Theorem 2.2 will be verified using the following lemma. Let (Top, $\times, *$ ) be the category of spaces (weak Hausdorff $k$-spaces) endowed with the Cartesian product and $\left(\mathrm{Top}_{*}, \wedge, S^{0}\right)$ the based spaces endowed with the smash product, where $S^{0}=\{0,1\}$ is the unit, with 0 as basepoint. Recall that the functor:

$$
\begin{aligned}
(-)_{+}:(\text {Top }, \times, *) & \longrightarrow\left(\text { Top }_{*}, \wedge, S^{0}\right), \\
X & \longmapsto X \coprod\{*\}
\end{aligned}
$$

is strong symmetric monoidal: $X_{+} \wedge Y_{+} \cong(X \times Y)_{+}$. Since (Top, $\left.\times, *\right)$ is a Cartesian symmetric monoidal category, every space $C$ has a unique comonoidal structure with respect to the Cartesian product, see [AM10, Example 1.19]. The counit $\varepsilon: C \rightarrow *$ is the unique map to the terminal object, and the comultiplication $\Delta: C \rightarrow C \times C$ is the diagonal $\Delta=\left(\mathrm{id}_{C}, \mathrm{id}_{C}\right)$. The comultiplication is always cocommutative. Since the functor $(-)_{+}$: Top $\rightarrow$ Top $_{*}$ is strong symmetric monoidal, it sends the cocommutative comonoid $(C, \Delta, \varepsilon)$ to a cocommutative comonoid $\left(C_{+}, \Delta_{+}, \varepsilon_{+}\right)$. The next result says that these are the only possible comonoids in $\left(\mathrm{Top}_{*}, \wedge, S^{0}\right)$. It turns out that this is purely a point-set argument, and the result remains valid for the category of sets, and simplicial sets, denoted respectively Set and sSet.

Lemma 2.4. Let the category $\mathcal{C}$ be either Set, sSet or Top endowed with its Cartesian symmetric monoidal structure. Then the faithful strong symmetric monoidal functor $(-)_{+}:(\mathcal{C}, \times, *) \rightarrow\left(\mathcal{C}_{*}, \wedge, S^{0}\right)$ lifts to an equivalence of categories $(-)_{+}: \operatorname{coMon}(\mathcal{C}) \rightarrow$ coMon $\left(\mathcal{C}_{*}\right)$. In particular, any comonoid in $\mathcal{C}_{*}$ is cocommutative and isomorphic to a certain comonoid $\left(C_{+}, \Delta_{+}, \varepsilon_{+}\right)$where $(C, \Delta, \varepsilon)$ is a cocommutative comonoid in $\mathcal{C}$ with the diagonal as a comultiplication.

Proof. We argue only for $\mathcal{C}=$ Set and claim the other cases are similar. The functor is 
clearly faithful. Let us first show that it is essentially surjective on comonoid objects. Let $\left(C^{\prime}, \Delta^{\prime}, \varepsilon^{\prime}\right)$ be a comonoid in Set $_{*}$. We first argue that $\Delta^{\prime}(c)=c \wedge c$ for all $c \neq *$ in $C$. Let us denote $\Delta^{\prime}(c)=c_{1} \wedge c_{2}$.

If $c_{1}=*$, then $\left(\mathrm{id}_{C}^{\prime} \wedge \varepsilon^{\prime}\right)\left(c_{1} \wedge c_{2}\right)=*$, as the map $\operatorname{id}_{C}^{\prime} \wedge \varepsilon^{\prime}$ is pointed, and thus counitality of $C^{\prime}$ shows:

$$
c=\left(\left(\mathrm{id}_{C}^{\prime} \wedge \varepsilon^{\prime}\right) \circ \Delta^{\prime}\right)(c)=\left(\mathrm{id}_{C}^{\prime} \wedge \varepsilon^{\prime}\right)\left(c_{1} \wedge c_{2}\right)=*,
$$

which is a contradiction with $c \neq *$. Thus $c_{1} \neq *$, and similarly one can show $c_{2} \neq *$ when $c \neq *$.

Let us show $\varepsilon^{\prime}\left(c_{1}\right)=1$ and $\varepsilon^{\prime}\left(c_{2}\right)=1$, when $c \neq *$. If we assume $\varepsilon^{\prime}\left(c_{2}\right)=0$, then we obtain again equation (2.1) which is a contradiction with $c \neq *$. Thus $\varepsilon^{\prime}\left(c_{2}\right) \neq 0$ and we prove similarly $\varepsilon^{\prime}\left(c_{1}\right) \neq 0$ when $c \neq *$.

Let us prove $\Delta(c)=c \wedge c$, for $c \neq *$. Since $C^{\prime}$ is counital, we get:

$$
c=\left(\left(\operatorname{id}_{C}^{\prime} \wedge \varepsilon^{\prime}\right) \circ \Delta^{\prime}\right)(c)=c_{1} \wedge \varepsilon^{\prime}\left(c_{2}\right)=c_{1} \wedge 1 .
$$

So $c_{1}=c$. Similarly, $c_{2}=c$.

Now notice that $C^{\prime} \cong \varepsilon^{\prime-1}(0) \coprod \varepsilon^{\prime-1}(1)$. Let $c \in \varepsilon^{\prime-1}(0)$. Then from the equation:

$$
\left(\left(\operatorname{id}_{C^{\prime}} \wedge \varepsilon^{\prime}\right) \circ \Delta^{\prime}\right)(c)=\left(\operatorname{id}_{C^{\prime}} \wedge \varepsilon^{\prime}\right)(c \wedge c)=c \wedge 0=*,
$$

counitality of $C^{\prime}$ concludes that $c=*$. Thus $\varepsilon^{\prime-1}(0)=*$. Regard $C=\varepsilon^{\prime-1}(1)$ as an object in Set. Denote the diagonal on $C$ by $\Delta=\left(\mathrm{id}_{C}, \mathrm{id}_{C}\right): C \rightarrow C \times C$ and $\varepsilon: C \rightarrow *$ the unique map to the point. We have just shown that $\left(C_{+}, \Delta_{+}, \varepsilon_{+}\right)$is isomorphic to the comonoid $\left(C^{\prime}, \Delta^{\prime}, \varepsilon^{\prime}\right)$ in $\operatorname{Set}_{*}$. This proves that the functor $(-)_{+}$is essentially surjective on comonoids.

Let us show the functor $(-)_{+}$: Set $\rightarrow$ Set $_{*}$ is full on comonoid objects. Given two comonoids denoted by $\left(C_{+},\left(\Delta_{C}\right)_{+},\left(\varepsilon_{C}\right)_{+}\right)$and $\left(D_{+},\left(\Delta_{D}\right)_{+},\left(\varepsilon_{D}\right)_{+}\right)$in Set $_{*}$, let $f: C_{+} \rightarrow D_{+}$be a map of comonoids in $\mathrm{Set}_{*}$. Then counitality gives the commutative diagram:

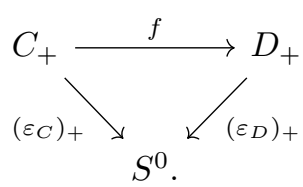

If, for $c \neq *$ in $C_{+}$, we have $f(c)=*$, then commutativity of the diagram gives:

$$
1=\left(\varepsilon_{C}\right)_{+}(c)=\left(\left(\varepsilon_{D}\right)_{+} \circ f\right)(c)=\left(\varepsilon_{D}\right)_{+}(*)=0,
$$

which is a contradiction. Thus the map $f: C_{+} \rightarrow D_{+}$is induced by a map $C \rightarrow D$ in Set, which proves that $(-)_{+}$: Set $\rightarrow$ Set $_{*}$ is full on comonoid objects.

Remark 2.5. Subsequently, we will only be using that all comonoids in the symmetric monoidal category $\left(\mathrm{Top}_{*}, \wedge, S^{0}\right)$ are cocommutative. Notice that Theorem 2.2 does not apply for the adjoint pair of functors:

$$
(-)_{+}:(\mathrm{Top}, \times, *) \rightleftarrows\left(\mathrm{Top}_{*}, \wedge, S^{0}\right): U,
$$

where $U:$ Top $_{*} \rightarrow$ Top is the forgetful functor, as $U$ does not respect condition (i) of Theorem 2.2.

Recall that given a commutative monoid $R$ in $\mathcal{C}$, the category of (left) modules 
over $R$ in $\mathcal{C}$, denoted $\operatorname{Mod}_{R}(\mathcal{C})$ is a symmetric monoidal category, where the unit is $R$ and the monoidal product is denoted $\otimes_{R}$ and is defined as the coequalizer:

$$
M \otimes R \otimes N \underset{\left(\alpha_{M} \circ \tau\right) \otimes \mathrm{id}_{N}}{\stackrel{\mathrm{id}_{M} \otimes \alpha_{N}}{\longrightarrow}} M \otimes N
$$

where $\alpha_{M}: R \otimes M \rightarrow M$ and $\alpha_{N}: R \otimes N \rightarrow N$ are the (left) $R$-actions on $M$ and $N$ respectively. This leads to the following definition.

Definition 2.6. Let $R$ be a commutative monoid in $\mathcal{C}$. A coalgebra $(C, \Delta, \varepsilon)$ over $R$ in $\mathcal{C}$, or an $R$-coalgebra in $\mathcal{C}$, is a comonoid $(C, \Delta, \varepsilon)$ in the symmetric monoidal category $\left(\operatorname{Mod}_{R}(\mathcal{C}), \otimes_{R}, R\right)$. A morphism $f:(C, \Delta, \varepsilon) \rightarrow\left(C^{\prime}, \Delta^{\prime}, \varepsilon^{\prime}\right)$ of $R$-coalgebras in $\mathcal{C}$ is a morphism of comonoids in $\operatorname{Mod}_{R}(\mathcal{C})$.

\section{Coalgebras in diagram spectra}

Let us recall the definitions from [MMSS01] and set the notation. Let $\mathcal{D}=$ $(\mathcal{D}, \otimes, 0)$ be a locally small symmetric monoidal based topological category with unit object 0 and continuous monoidal product $\otimes$, with base point $*$. Let Top $*$ be the category of based spaces (weak Hausdorff $k$-spaces). Recall that a $\mathcal{D}$-space $X$ is a continuous based functor $X: \mathcal{D} \rightarrow \operatorname{Top}_{*}$. If $X$ and $Y$ are $\mathcal{D}$-spaces, their (internal) smash product $X \wedge Y$ is a $\mathcal{D}$-space such that, for each object $d$ in $\mathcal{D}$, we have:

$$
(X \wedge Y)(d)=\operatorname{colim}_{e \otimes f \rightarrow d}(X(e) \wedge Y(f)),
$$

where the colimit is taken over the commutative triangles:

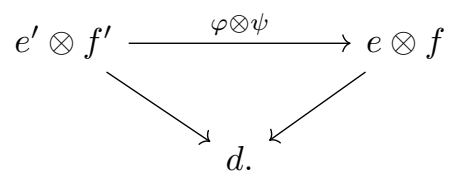

As $\mathcal{D}$ is locally small, we can interpret the above colimit as a coend, i.e., $(X \wedge Y)(d)$ is the following coequalizer in $\mathrm{Top}_{*}$ :

$$
\bigvee_{\substack{(\varphi, \psi) \text { in } \\ \operatorname{Mor}(\mathcal{D} \times \mathcal{D})}} \mathcal{D}(e \otimes f, d) \wedge X\left(e^{\prime}\right) \wedge Y\left(f^{\prime}\right) \frac{(\varphi \otimes \psi)^{*} \wedge \mathrm{id} \wedge \text { id }}{i \operatorname{id\wedge X(\varphi )\wedge Y(\psi )}} \underset{\substack{(e, f) \text { in } \\ \mathrm{Ob}(\mathcal{D} \times \mathcal{D})}}{\longrightarrow} \mathcal{D}(e \otimes f, d) \wedge X(e) \wedge Y(f)
$$

See more detail in [MMSS01, Definition 21.4]. Then $\mathcal{D}$-spaces form a symmetric monoidal category denoted $\operatorname{Top}_{*}^{\mathcal{D}}$ (see [MMSS01, Theorem 1.7] where the category is denoted $\mathcal{D} \mathcal{T}$ ).

A commutative monoid in $\operatorname{Top}_{*}^{\mathcal{D}}$ is a lax symmetric monoidal functor $\mathcal{D} \rightarrow$ Top $_{*}$. Let $R$ be a commutative monoid in Top $_{*}^{\mathcal{D}}$ with unit $\lambda: S^{0} \rightarrow R(0)$, and product $\phi: R(d) \wedge R(e) \rightarrow R(d \otimes e)$, for any $d$ and $e$ in $\mathcal{D}$. A $\mathcal{D}$-spectrum $X$ over $R$ is an $R$-module in $\operatorname{Top}_{*}^{\mathcal{D}}$. It is a $\mathcal{D}$-space $X: \mathcal{D} \rightarrow$ Top $_{*}$ together with continuous maps $\sigma: R(d) \wedge X(e) \rightarrow X(d \otimes e)$, natural in $d$ and $e$, such that the composite:

$$
X(d) \cong S^{0} \wedge X(d) \stackrel{\lambda \wedge \text { id }}{\longrightarrow} R(0) \wedge X(d) \longrightarrow X(0 \otimes d) \cong X(d)
$$


is the identity and the following diagram commutes:

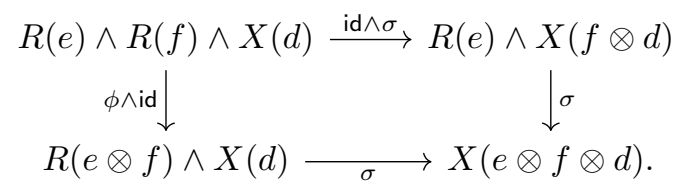

As recalled in the previous section, the smash product $X \wedge_{R} Y$ of two $R$-modules $X$ and $Y$ is defined as the coequalizer:

$$
X \wedge R \wedge Y \underset{\left(\sigma_{X} \circ \tau\right) \wedge \text { id }_{Y}}{\stackrel{\mathrm{id}_{X} \wedge \sigma_{Y}}{\longrightarrow}} X \wedge Y
$$

where $\tau$ is the twist isomorphism. We denote the category of $R$-modules $\operatorname{Mod}_{R}\left(\operatorname{Top}_{*}^{\mathcal{D}}\right)$ simply by $\mathrm{Sp}_{R}^{\mathcal{D}}$. Subsequently we assume the following conditions on the topological category $\mathcal{D}$.

Property 3.1. There is a faithful strong symmetric monoidal continuous based functor $\mathbb{S}: \mathcal{D} \rightarrow$ Top $_{*}$.

Property 3.2. If $\mathbb{S}(d)$ is homeomorphic to the base point in $\mathrm{Top}_{*}$, then $d$ is isomorphic to the base point $*$ in $\mathcal{D}$. If $\mathbb{S}(d)$ is homeomorphic to $S^{0}$, then $d$ is isomorphic to the unit object 0 in $\mathcal{D}$.

Example 3.3. Recall, from [MMSS01, Examples 4.2, 4.4, 4.6, 4.8], that particular choices of the category $\mathcal{D}$ recover the usual definition of the categories of symmetric spectra, orthogonal spectra, $\Gamma$-spaces and $\mathcal{W}$-spaces, among others. It is also shown that there exist faithful strong symmetric monoidal based functors $\mathbb{S}: \mathcal{D} \rightarrow$ Top $_{*}$ for each of the choices of $\mathcal{D}$, which of course identify with the usual sphere spectrum definition. It is elementary to check that Property 3.2 is verified in each of these cases. There may be other examples of interest.

We now state the main theorem.

Theorem 3.4. Let $\mathcal{D}$ satisfy Properties 3.1 and 3.2. Let $(R, \phi, \lambda)$ be a commutative monoid in $\operatorname{Top}_{*}^{\mathcal{D}}$, where $R(0) \cong S^{0}$. Let $(C, \Delta, \varepsilon)$ be an $R$-coalgebra in $\operatorname{Top}_{*}^{\mathcal{D}}$. Then $C$ is a cocommutative $R$-coalgebra. In particular, all coalgebras over the sphere spectrum in symmetric spectra, orthogonal spectra, $\Gamma$-spaces and $\mathcal{W}$-spaces are cocommutative.

Remark 3.5. We show here that, in symmetric spectra or orthogonal spectra, any cofibration of commutative $\mathbb{S}$-algebras is the identity at level 0 . For a cofibrant object $R$, the unit map $\mathbb{S} \rightarrow R$ is a cofibration and hence it follows that $R(0) \cong \mathbb{S}(0)=S^{0}$. Thus, in symmetric spectra or orthogonal spectra, any cofibrant commutative $\mathbb{S}$ algebra $R$ has the property that $R(0) \cong S^{0}$.

Recall the free commutative $\mathbb{S}$-algebra functor, denoted $\mathbb{C}(X)=\bigvee_{n \geqslant 0} X^{\wedge n} / \Sigma_{n}$, with $X^{\wedge 0}=\mathbb{S}$. The cofibrations in the model structure on commutative $\mathbb{S}$-algebras from [MMSS01, 15.1] are built by applying $\mathbb{C}$ to the positive cofibrations defined in [MMSS01, 14.1]. There it is noted that these positive cofibrations are homeomorphisms at level 0 . In fact, by [MMSS01, 6.2], any generating positive cofibration is of the form $F_{k} i$ with $i$ an $h$-cofibration and $k>0$. Since $k>0$, level 0 of these maps is 
the identity map on the trivial one point space $*$. Since $X \wedge n(0) \cong X(0) \wedge n$, the only contribution to level 0 for the map $\mathbb{C} F_{k} i$ is the identity map on $S^{0}$ coming from the summand with $n=0$. Thus, any cofibration of commutative $\mathbb{S}$-algebras is the identity on level 0 .

This also holds in the $\mathbb{S}$-model structure from [Shi04, 3.2] for commutative $\mathbb{S}$ algebras. There a cofibration of commutative $\mathbb{S}$-algebras is shown to be an underlying positive $\mathbb{S}$-cofibration of $\mathbb{S}$-modules by $[\mathbf{S h i 0 4}, 4.1]$. These maps are isomorphisms in level 0 by definition [Shi04, Section 3]. Elsewhere these model structures are referred to as the "flat" or "positive flat" model structures [Sch].

We wish to prove Theorem 3.4. We will use Theorem 2.2 for the following adjoint pair of functors. Given a commutative monoid $R$ in $\operatorname{Top}_{*}^{\mathcal{D}}$, the free $R$-module functor is the left adjoint to the evaluation at the unit object 0 of $\mathcal{D}$ (see [MMSS01, Definition 1.3]):

$$
R \wedge-: \mathrm{Top}_{*} \rightleftarrows \mathrm{Sp}_{R}^{\mathcal{D}}: \mathrm{Ev}_{0} .
$$

Equation (3.1) shows that there is a natural map $X(0) \wedge Y(0) \rightarrow\left(X \wedge_{R} Y\right)(0)$, for any $X$ and $Y$ in $\mathrm{Sp}_{R}^{\mathcal{D}}$, which makes the functor $\mathrm{Ev}_{0}: \mathrm{Sp}_{R}^{\mathcal{D}} \rightarrow \mathrm{Top}_{*}$ lax symmetric monoidal. We have already shown in Lemma 2.4 that condition (ii) of Theorem 2.2 is verified by $\left(\mathrm{Top}_{*}, \wedge, S^{0}\right)$. So we only need to know when conditions (i) and (iii) of Theorem 2.2 are verified.

Let us first investigate condition (iii) of Theorem 2.2. Notice that a map $f: X \rightarrow Y$ in $\operatorname{Top}_{*}^{\mathcal{D}}$ is an epimorphism if and only if, for any object $d$ in $\mathcal{D}$, the map $f: X(d) \rightarrow$ $Y(d)$ is an epimorphism in $\mathrm{Top}_{*}$, i.e. a surjective based continuous map.

Lemma 3.6. Let $\mathcal{D}$ satisfy Properties 3.1 and 3.2. Let $(R, \phi, \lambda)$ be a commutative monoid in $\operatorname{Top}_{*}^{\mathcal{D}}$. Let $(C, \Delta, \varepsilon)$ be an $R$-coalgebra in $\mathrm{Top}_{*}^{\mathcal{D}}$. Then the natural map $R \wedge C(0) \longrightarrow C$ is an epimorphism of $R$-modules in Top ${ }_{*}^{\mathcal{D}}$.

The main idea of the proof is to look at the consequences of counitality of an $R$ coalgebra $C$ with respect to the identifications in the smash product $C \wedge_{R} C$. Before proving the lemma, we need the following result.

Lemma 3.7. Let $X$ be an object of $\left(\mathrm{Top}_{*}, \wedge, S^{0}\right)$.

(i) If we are given pointed maps $f: S^{0} \rightarrow X$ and $g: X \rightarrow S^{0}$ such that the induced map:

$$
S^{0} \wedge X \underset{f \wedge g}{\stackrel{f}{\cong}} X \wedge S^{0}
$$

is an isomorphism, then either $X \cong *$ and the morphisms $f$ and $g$ are the trivial maps, or $X \cong S^{0}$ and $f$ and $g$ are isomorphisms.

(ii) Suppose we are given pointed sets $Y$ and $Z$ together with pointed maps $f: S^{0} \rightarrow$ $Y, g: X \rightarrow Z, f^{\prime}: X \rightarrow Y$ and $g^{\prime}: S^{0} \rightarrow Z$ such that the following diagram commutes:

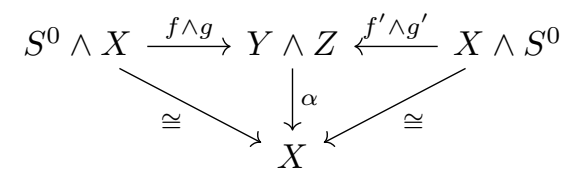

for a pointed map $\alpha: Y \wedge Z \rightarrow X$. Then either $X \cong *$ and the morphisms $g$ and 
$f^{\prime}$ are trivial, or $X \cong S^{0}$ and the composite $S^{0} \cong S^{0} \wedge S^{0} \stackrel{f \wedge g^{\prime}}{\longrightarrow} Y \wedge Z \stackrel{\alpha}{\rightarrow} X$ is an isomorphism.

Proof. The proof is purely a point set argument. For (i), assume $X \not *$. Let $x \neq *$ in $X$. The commutativity of the diagram:

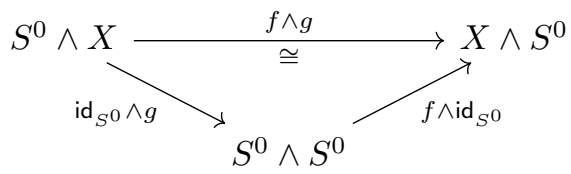

implies that $f \wedge \mathrm{id}_{S^{0}}$ is surjective. Thus any element $x \wedge 1$ in $X \wedge S^{0}$ is of the form $f(1) \wedge 1$. This implies $X \cong S^{0}$.

For (ii), assume again $X \not * *$. The map $\alpha$ is surjective as $\alpha \circ(f \wedge g)$ is an isomorphism. For an element $x \neq *$ in $X$, denote by $y \wedge z$ an element in $Y \wedge Z$ such that $\alpha(y \wedge z)=x$. Then we get:

$$
f(1) \wedge g(x)=y \wedge z=f^{\prime}(x) \wedge g^{\prime}(1) .
$$

Thus $y=f(1)=f^{\prime}(x)$ and $z=g^{\prime}(1)=g(x)$. The desired composite is an isomorphism. Whence $X \cong S^{0}$.

Proof of Lemma 3.6. We need to prove that the continuous structure map $\sigma: R(d) \wedge$ $C(0) \rightarrow C(d)$ is surjective for each $d$ in $\mathcal{D}$. If $d=0$, since the composition $C(0) \cong$ $S^{0} \wedge C(0) \longrightarrow R(0) \wedge C(0) \stackrel{\sigma}{\longrightarrow} C(0)$ is the identity, then $\sigma: R(0) \wedge C(0) \rightarrow C(0)$ is surjective. If $d=*$, then the map $\sigma: R(*) \wedge C(0) \longrightarrow C(*)$ is trivial, as the functors $C$ and $R$ are pointed.

Let us assume now that $d$ is an object in $\mathcal{D}$, where $d \not 0, *$. The above definition of the smash product of $R$-modules in Top ${ }_{*}^{\mathcal{D}}$ leads to the explicit definition of $C \wedge_{R} C$ as a quotient space of:

$$
\left(C \wedge_{R} C\right)(d)=\left(\bigvee_{(e, f) \in \mathcal{D} \times \mathcal{D}} \mathcal{D}(e \otimes f, d) \wedge C(e) \wedge C(f)\right) / \sim_{R}
$$

for any $d$ in $\mathcal{D}$. The relations here are induced by the internal smash product (see coequalizer (3.1) for $X=Y=C$ ) and by the $R$-action via the structure maps $\sigma: R(e) \wedge C(f) \rightarrow C(e \otimes f)$. Notice that the natural isomorphisms $d \otimes 0 \cong$ $0 \otimes d \stackrel{\cong}{\rightrightarrows} d$ imply that there is at least one copy of $C(d) \wedge C(0)$ and $C(0) \wedge C(d)$ in $\left(C \wedge \wedge_{R} C\right)(d)$.

First let us show that an element in $C(d) \wedge C(0)$ is not identified with an element in $C(0) \wedge C(d)$ in $(C \wedge C)(d)$ via the coequalizer (3.1). For this matter, assume there are objects $e$ and $f$ in $\mathcal{D}$ that fit in either of the following commutative diagrams in $\mathcal{D}$ :
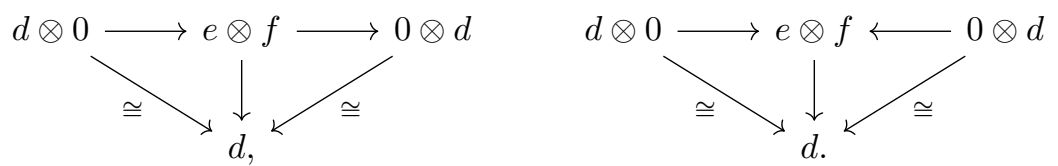

Using Property 3.1, we apply the functor $\mathbb{S}: \mathcal{D} \rightarrow$ Top $_{*}$ and get the commutative 
diagrams in $\mathrm{Top}_{*}$ :
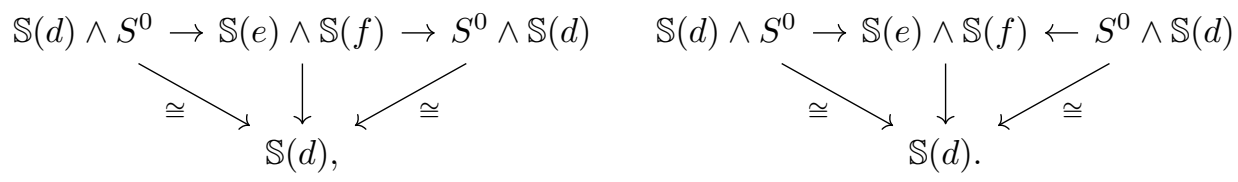

Then, using Lemma 3.7 and Property 3.2 , we get either $d \cong 0$ or $d \cong *$ in both cases, which is a contradiction. Thus elements in $C(d) \wedge C(0)$ are not identified with elements in $C(0) \wedge C(d)$ in $(C \wedge C)(d)$.

Now we consider $C \wedge_{R} C$ instead of $C \wedge C$. Some identifications do occur in the pointed space $\left(C \wedge_{R} C\right)(d)$ via the $R$-action structure maps. Recall that since $C$ is counital, we have the commutative diagram:

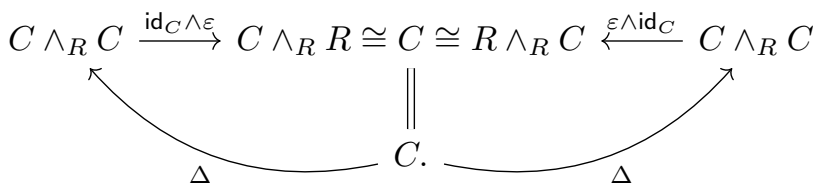

The commutativity shows that the maps $\varepsilon \wedge \mathrm{id}_{C}$ and $\mathrm{id}_{C} \wedge \varepsilon$ are epimorphisms in $\mathrm{Top}_{*}^{\mathcal{D}}$, hence surjective once evaluated at $d$. Suppose now that the structure map $\sigma: R(d) \wedge C(0) \rightarrow C(d)$ is not surjective. Consider an element $c$ in $C(d)$ not in the image of $\sigma$. On the one hand, we can view $C$ as $C \wedge_{R} R$ and so, if we consider $c$ in $C(d)$ as $c \wedge \lambda(1)$ in $C(d) \wedge R(0)$ of $\left(C \wedge_{R} R\right)(d)$, then we can take its preimage under the map:

$$
\operatorname{id}_{C} \wedge \varepsilon:\left(C \wedge_{R} C\right)(d) \longrightarrow\left(C \wedge_{R} R\right)(d) .
$$

The preimage lies in the contribution from $C(d) \wedge C(0)$ in (3.2). On the other hand, if we view $C$ as $R \wedge_{R} C$, consider $c$ in $C(d)$ as $\lambda(1) \wedge c$ in $R(0) \wedge C(d)$ of $\left(R \wedge_{R} C\right)(d)$ and take its preimage under the map:

$$
\varepsilon \wedge \mathrm{id}_{C}:\left(C \wedge_{R} C\right)(d) \longrightarrow\left(R \wedge_{R} C\right)(d),
$$

it belongs to the copy $C(0) \wedge C(d)$ in (3.2). Since we have supposed that $\sigma$ is not surjective and that $c$ does not belong to the image of $\sigma$ in $C(d)$, we get that the two preimages $\left(\operatorname{id}_{C} \wedge \varepsilon\right)^{-1}(c)$ and $\left(\varepsilon \wedge \mathrm{id}_{C}\right)^{-1}(c)$ in $\left(C \wedge_{R} C\right)(d)$ are disjoint from each other. But then the commutativity of diagram (3.3) forces two different values of $\Delta(c)$ for the map $\Delta: C \longrightarrow C \wedge_{R} C$. We get a contradiction, thus $\sigma: R(d) \wedge C(0) \rightarrow C(d)$ must be surjective.

Remark 3.8. Notice that we did not require $R(0) \cong S^{0}$ for Lemma 3.6. It is valid for any commutative monoid $R$ in $\operatorname{Top}_{*}^{\mathcal{D}}$. This suggests that, even though $R$-coalgebras in $\operatorname{Top}_{*}^{\mathcal{D}}$ are not necessarily cocommutative when $R(0) \varsubsetneqq S^{0}$, there are restrictions on the possibilities of $R$-coalgebras in $\operatorname{Top}_{*}^{\mathcal{D}}$.

Remark 3.9. In the proof of Lemma 3.6 above, choosing a particular category $\mathcal{D}$ might simplify the reader's understanding. For instance, if we choose $\mathcal{D}=\Sigma_{+}$as in [MMSS01, Example 4.2], where $\Sigma$ is the category of finite sets and their permutations, we obtain the usual category of symmetric spectra $\mathrm{Sp}^{\Sigma}:=\mathrm{Sp}_{\mathbb{S}}^{\Sigma_{+}}$as in [HSS00]. Lemma 3.6, for $\mathbb{S}$-coalgebras in $\mathrm{Sp}^{\Sigma}$, appeared in an early version of [HS] before being 
developed further here. Equation (3.1) of the internal smash product of $\Sigma_{+}$-spaces $X$ and $Y$ at the $n$th level simplifies to:

$$
(X \wedge Y)_{n}=\bigvee_{p+q=n} \Sigma_{n}^{+} \wedge_{\Sigma_{p} \times \Sigma_{q}} X_{p} \wedge Y_{q}
$$

where $\Sigma_{n}$ is the symmetric group on $n$ letters. Thus we see directly that the copies $X_{n} \wedge Y_{0}$ and $X_{0} \wedge Y_{n}$ are never identified in the internal smash product, but they can be when considering $\left(X \wedge_{\mathbb{S}} Y\right)_{n}$. In particular, we do not need Lemma 3.7 here.

In order to prove Theorem 3.4, we now investigate condition (i) of Theorem 2.2 with the functor $\mathrm{Ev}_{0}: \mathrm{Sp}_{R}^{\mathcal{D}} \rightarrow \mathrm{Top}_{*}$. We need the next two results.

Lemma 3.10. Let $X$ and $Y$ be pointed spaces. For any commutative monoid $(R, \phi, \lambda)$ in $\operatorname{Top}_{*}^{\mathcal{D}}$, and any object $d$ in $\mathcal{D}$, we have a homeomorphism of pointed spaces:

$$
R(d) \wedge X \wedge Y \cong\left[(R \wedge X) \wedge_{R}(R \wedge Y)\right](d) .
$$

Proof. The proof follows by adjointness of the functor $R \wedge-: \mathrm{Top}_{*} \rightarrow \mathrm{Sp}_{R}^{\mathcal{D}}$ as in [MMSS01, Lemma 21.3].

Lemma 3.11. Let $\mathcal{D}$ satisfy Properties 3.1 and 3.2 . Let $(R, \phi, \lambda)$ be a commutative monoid in $\operatorname{Top}_{*}^{\mathcal{D}}$, where $R(0) \cong S^{0}$. Let $(C, \Delta, \varepsilon)$ be an $R$-coalgebra in $\mathrm{Top}_{*}^{\mathcal{D}}$. Then there is a homeomorphism of pointed spaces:

$$
C(0) \wedge C(0) \cong\left(C \wedge_{R} C\right)(0) \text {. }
$$

Remark 3.12. The result of Lemma 3.11 is automatic in most categories of interest, and does not require $C$ to be a coalgebra. Indeed, we have $(X \wedge Y)(0) \cong X(0) \wedge Y(0)$ for any symmetric spectra or orthogonal spectra $X$ and $Y$. In other words, the functor $\mathrm{Ev}_{0}: \mathrm{Sp}_{R}^{\mathcal{D}} \rightarrow$ Top $_{*}$ is strong symmetric monoidal when $R(0) \cong S^{0}$. But this is not true in general (for instance in $\mathcal{W}$-spaces and $\Gamma$-spaces, see Examples 3.14 and 3.16 below).

Proof. Denote by $\sigma: R \wedge C(0) \rightarrow C$ the natural epimorphic map of Lemma 3.6. Let us consider the map of $R$-modules $\sigma \wedge \sigma:(R \wedge C(0)) \wedge_{R}(R \wedge C(0)) \rightarrow C \wedge_{R} C$. If we evaluate on the unit object 0 we get a map:

$$
\sigma \wedge \sigma: C(0) \wedge C(0) \cong\left[(R \wedge C(0)) \wedge_{R}(R \wedge C(0))\right](0) \longrightarrow\left(C \wedge_{R} C\right)(0),
$$

where the left homeomorphism is induced by Lemma 3.10 and $R(0) \cong S^{0}$. Recall that $\left(C \wedge_{R} C\right)(0)$ is obtained from $(C \wedge C)(0)$ by coequalizing the $R$-action, so that we get a surjective continuous pointed map $(C \wedge C)(0) \rightarrow\left(C \wedge_{R} C\right)(0)$. The map $\sigma \wedge \sigma$ factors through the space $(C \wedge C)(0)$ :

$$
\left[(R \wedge C(0)) \wedge_{R}(R \wedge C(0))\right](0) \stackrel{\sigma \wedge \sigma}{\longrightarrow}(C \wedge C)(0)
$$

Recall that $(C \wedge C)(0)=(\bigvee \mathcal{D}(e \otimes f, 0) \wedge C(e) \wedge C(f)) / \sim$. From Lemma 3.6, given an element $\alpha \wedge c_{1} \wedge c_{2}$ in $\mathcal{D}(e \otimes f, 0) \wedge C(e) \wedge C(f)$, there exists $r_{1} \in R(e), r_{2} \in R(f)$, $c_{1}^{\prime}, c_{2}^{\prime} \in C(0)$ such that $\sigma\left(r_{1} \wedge c_{1}^{\prime}\right)=c_{1}$ and $\sigma\left(r_{2} \wedge c_{2}^{\prime}\right)=c_{2}$. Hence $(\sigma \wedge \sigma)\left(\alpha \wedge r_{1} \wedge\right.$ $\left.c_{1}^{\prime} \wedge r_{2} \wedge c_{2}^{\prime}\right)=\alpha \wedge c_{1} \wedge c_{2}$. Thus the map $\sigma \wedge \sigma$ is surjective. 
Next we show that the map $\sigma \wedge \sigma: C(0) \wedge C(0) \rightarrow\left(C \wedge_{R} C\right)(0)$ is injective. Explicitly, the map $\sigma \wedge \sigma$ sends $C(0) \wedge C(0)$ to the copy $\mathcal{D}(0 \otimes 0,0) \wedge C(0) \wedge C(0)$ in $\left(C \wedge_{R}\right.$ $C)(0)$ via the natural isomorphism $0 \otimes 0 \stackrel{\cong}{\longrightarrow} 0$. Since $C$ is counital, we can consider the maps of $R$-modules:

$$
C \wedge_{R} C \stackrel{\mathrm{id}_{C} \wedge \varepsilon}{\longrightarrow} C \wedge_{R} R \cong C, \quad C \wedge_{R} C \stackrel{\varepsilon \wedge^{\mathrm{id}_{C}}}{\longrightarrow} R \wedge_{R} C \cong C .
$$

Evaluating again at 0 and factoring the above maps through the product, we get a continuous pointed map:

$$
\left(C \wedge_{R} C\right)(0) \stackrel{\left(\mathrm{id}_{C} \wedge \varepsilon\right) \wedge\left(\varepsilon \wedge \text { id }_{C}\right)}{\longrightarrow} C(0) \wedge C(0) .
$$

Explicitly, the above map acts as the identity on the copy of $C(0) \wedge C(0)$ in $\left(C \wedge_{R}\right.$ $C)(0)$. That is, the composite:

$$
C(0) \wedge C(0) \stackrel{\sigma \wedge \sigma}{\longrightarrow}\left(C \wedge_{R} C\right)(0) \stackrel{\left(\mathrm{id}_{C} \wedge \varepsilon\right) \wedge\left(\varepsilon \wedge \mathrm{id}_{C}\right)}{\longrightarrow} C(0) \wedge C(0)
$$

is the identity by the counital property. Thus $\sigma \wedge \sigma$ is injective. Therefore $\sigma \wedge \sigma$ induces the desired homeomorphism with inverse $\left(\operatorname{id}_{C} \wedge \varepsilon\right) \wedge\left(\varepsilon \wedge \mathrm{id}_{C}\right)$.

Proof of Theorem 3.4. We apply Theorem 2.2 to the adjoint pair of functors:

$$
R \wedge-: \operatorname{Top}_{*} \rightleftarrows \mathrm{Sp}_{R}^{\mathcal{D}}: \mathrm{Ev}_{0} .
$$

Lemma 3.11 proves (i). Lemma 2.4 shows (ii). Finally, Lemma 3.6 induces (iii).

We end this section with three examples.

Example 3.13. The functor $R \wedge-: \mathrm{Top}_{*} \rightarrow \mathrm{Sp}_{R}^{\mathcal{D}}$ does not lift to an essentially surjective functor on comonoid objects. For instance, when $R$ is the sphere spectrum $\mathbb{S}$ there exist examples of $\mathbb{S}$-coalgebras that are not isomorphic to suspension spectra. For example, in symmetric spectra, given a space $Y$ and a quotient space $Y / B$, one can form a counital coalgebra $C$ with $C_{0}=Y_{+}$, and $C_{n}=S^{n} \wedge(Y / B)_{+}$for $n>0$. The counit map on level $n$, that is, $S^{n} \wedge(Y / B)_{+} \rightarrow S^{n}$, is induced by the map from $Y / B_{+} \rightarrow S^{0}$ that sends only the base point to the base point of $S^{0}$.

Example 3.14. Let $\left(\mathcal{W}, \wedge, S^{0}\right)$ be the category of based spaces homeomorphic to finite CW complexes endowed with the usual smash product of spaces, as described in [MMSS01, Example 4.6]. The sphere spectrum $\mathbb{S}: \mathcal{W} \rightarrow$ Top $_{*}$ is defined as the strong symmetric monoidal faithful functor induced by inclusion. Recall from [MMSS01, Lemma 4.9] that any $\mathcal{W}$-space has a unique structure of $\mathcal{W}$-spectrum over $\mathbb{S}$. Moreover, for any $\mathcal{W}$-spaces $X$ and $Y$, we have $X \wedge Y \cong X \wedge_{\mathbb{S}} Y$. We provide here an example where:

$$
X\left(S^{0}\right) \wedge Y\left(S^{0}\right) \nsucceq(X \wedge Y)\left(S^{0}\right),
$$

for some choice of $\mathcal{W}$-spaces $X$ and $Y$. Recall from equation (3.1) that we have:

$$
(X \wedge Y)\left(S^{0}\right)=\left(\bigvee_{(K, L) \in \mathcal{W} \times \mathcal{W}} \mathcal{W}\left(K \wedge L, S^{0}\right) \wedge X(K) \wedge Y(L)\right) / \sim
$$


where elements $\alpha \wedge x_{K} \wedge y_{L}$ in $\mathcal{W}\left(K \wedge L, S^{0}\right) \wedge X(K) \wedge Y(Y)$ are identified with elements $x_{0} \wedge y_{0}$ in $X\left(S^{0}\right) \wedge Y\left(S^{0}\right) \cong \mathcal{W}\left(S^{0} \wedge S^{0}, S^{0}\right) \wedge X\left(S^{0}\right) \wedge Y\left(S^{0}\right)$ if and only if either one of the following type of identifications occurs:

(1) there exist maps $f: S^{0} \rightarrow K$ and $g: S^{0} \rightarrow L$ in $\mathcal{W}$ such that $X(f)\left(x_{0}\right)=x_{K}$, $Y(g)\left(y_{0}\right)=y_{L}$ and the following diagram commutes:

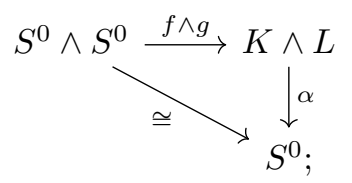

(2) there exist maps $f: K \rightarrow S^{0}$ and $g: L \rightarrow S^{0}$ in $\mathcal{W}$ such that $X(f)\left(x_{K}\right)=x_{0}$, $Y(g)\left(y_{L}\right)=y_{0}$ and the following diagram commutes:

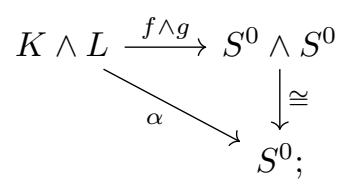

(3) there exist spaces $K^{\prime}$ and $L^{\prime}$ in $\mathcal{W}$, together with a map $\beta: K^{\prime} \wedge L^{\prime} \rightarrow S^{0}$ in $\mathcal{W}$, such that there exist maps $f: S^{0} \rightarrow K, g: S^{0} \rightarrow L, f^{\prime}: K \rightarrow K^{\prime}$ and $g^{\prime}: L \rightarrow L^{\prime}$ in $\mathcal{W}$ where $X(f)\left(x_{0}\right)=X\left(f^{\prime}\right)\left(x_{K}\right), Y(g)\left(y_{0}\right)=Y\left(g^{\prime}\right)\left(y_{L}\right)$ and the following diagram commutes:

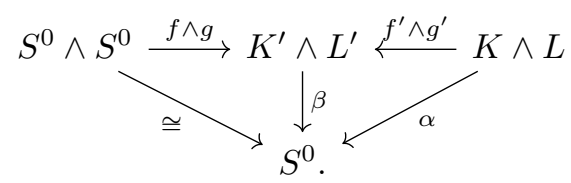

For any based space $A$, let us defined the infinite symmetric product of $A$, denoted $\mathrm{SP}(A)$, to be the free commutative monoid generated by $A$ in $\left(\mathrm{Top}_{*}, \times, *\right)$, see more details in [AGP02, Definition 5.2.1]. Write $\left[a_{1}, a_{2}, \ldots, a_{n}\right]$ for the equivalence class in $\operatorname{SP}(A)$ of the point $\left(a_{1}, a_{2}, \ldots, a_{n}, *, *, \ldots\right)$ in $\prod_{n \geqslant 1} A$. This defines a functor $\mathrm{SP}: \mathrm{Top}_{*} \rightarrow$ Top $_{*}$ where a map $f: A \rightarrow B$ induces $\mathrm{SP}(f): \mathrm{SP}(A) \rightarrow \mathrm{SP}(B)$ defined by $f\left(\left[a_{1}, \ldots, a_{n}\right]\right)=\left[f\left(a_{1}\right), \ldots, f\left(a_{n}\right)\right]$. We precompose by the functor $\mathbb{S}: \mathcal{W} \rightarrow$ Top $_{*}$ to obtain a based, continuous functor SP: $\mathcal{W} \rightarrow$ Top $_{*}$, which makes the infinite symmetric product SP into a $\mathcal{W}$-space. A standard result of Dold-Thom gives that, for $n \geqslant 1$, the spaces $\operatorname{SP}\left(S^{n}\right)$ are the Eilenberg-Mac Lane spaces $K(\mathbb{Z}, n)$, see [AGP02, Proposition 6.1.2], and thus SP is the Eilenberg-Mac Lane spectrum $H \mathbb{Z}$ in $\mathcal{W}$-spaces.

We argue here that $(\mathrm{SP} \wedge \mathrm{SP})\left(S^{0}\right) \not \mathrm{SP}\left(S^{0}\right) \wedge \mathrm{SP}\left(S^{0}\right)$. Let us describe particular elements $\alpha \wedge x_{K} \wedge y_{L}$ in $\mathcal{W}\left(K \wedge L, S^{0}\right) \wedge \mathrm{SP}(K) \wedge \mathrm{SP}(L)$, for particular $K$ and $L$ in $\mathcal{W}$, that are not identified to any element $x_{0} \wedge y_{0}$ in $\operatorname{SP}\left(S^{0}\right) \wedge \mathrm{SP}\left(S^{0}\right)$, via any identifications of the form (1), (2) and (3) above. Let us first describe $K$ and $L$ and the map $\alpha: K \wedge L \rightarrow S^{0}$. For the map $\alpha$ not be to be trivial, we choose $K$ and $L$ disconnected as follows. Let $K=\left\{*, k_{1}, k_{2}\right\}$ and $L=\left\{*, \ell_{1}, \ell_{2}\right\}$ be discrete spaces. Then the smash product $K \wedge L$ is given by the discrete space $\left\{*, k_{1} \wedge \ell_{1}, k_{1} \wedge \ell_{2}, k_{2} \wedge \ell_{1}, k_{2} \wedge \ell_{2}\right\}$. We define the map $\alpha: K \wedge L \rightarrow S^{0}$ by $\alpha(*)=0$ and:

$$
\alpha\left(k_{1} \wedge \ell_{1}\right)=0, \quad \alpha\left(k_{1} \wedge \ell_{2}\right)=1, \quad \alpha\left(k_{2} \wedge \ell_{1}\right)=1, \quad \alpha\left(k_{2} \wedge \ell_{2}\right)=0 .
$$

Given our choice of $\alpha: K \wedge L \rightarrow S^{0}$, the reader can verify that there are no maps 
$f: K \rightarrow S^{0}$ and $g: L \rightarrow S^{0}$ in $\mathcal{W}$ such that they fit in the commutative diagram:

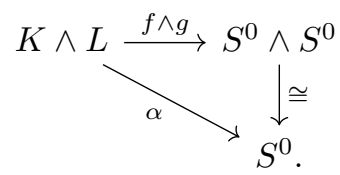

This shows that for any choice of $x_{K}$ and $y_{L}$ in $\operatorname{SP}(K)$ and $\operatorname{SP}(L)$, no element $\alpha \wedge$ $x_{K} \wedge y_{L}$ is identified with an element $x_{0} \wedge y_{0} \in \mathrm{SP}\left(S^{0}\right) \wedge \mathrm{SP}\left(S^{0}\right)$ in the identification of type (2) as described above.

Let $x_{K}=\left[k_{1}, k_{2}\right]$. Let $y_{L}$ be any non-basepoint element of $\operatorname{SP}(L)$. Any element of $\mathrm{SP}\left(S^{0}\right)$ is of the form $[1, \ldots, 1]$ and, for a map $f: S^{0} \rightarrow K$, the map $\mathrm{SP}(f)$ sends this element to $[f(1), \ldots, f(1)]$ in $\mathrm{SP}(K)$. Since $k_{1} \neq k_{2}$, there is no map $f: S^{0} \rightarrow K$ such that $\mathrm{SP}(f)\left(x_{0}\right)=x_{K}$ for some $x_{0} \in \mathrm{SP}\left(S^{0}\right)$. Thus, identifications of type (1) do not occur on the element $\alpha \wedge x_{K} \wedge y_{L}$ in $\mathcal{W}\left(K \wedge L, S^{0}\right) \wedge \mathrm{SP}(K) \wedge \mathrm{SP}(L)$.

Suppose now there exist objects $K^{\prime}$ and $L^{\prime}$ in $\mathcal{W}$, together with maps $f^{\prime}: K \rightarrow K^{\prime}$, $g^{\prime}: L \rightarrow L^{\prime}$ and $\beta: K^{\prime} \wedge L^{\prime} \rightarrow S^{0}$ in $\mathcal{W}$, such that the following diagram commutes:

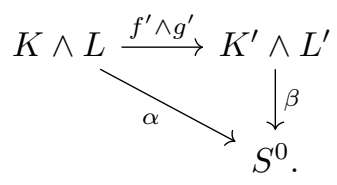

Since $\alpha$ is non-trivial, it follows that $K^{\prime} \wedge L^{\prime}$ is disconnected and $\beta$ is non-trivial. Since $K^{\prime} \wedge L^{\prime}$ is disconnected, if follows that both $K^{\prime}$ and $L^{\prime}$ are disconnected as well. We argue that the images $f^{\prime}\left(k_{1}\right)$ and $f^{\prime}\left(k_{2}\right)$ do not lie in the same path-component of $K^{\prime}$. If we suppose they are, then commutativity of the above diagram gives:

$$
0=\alpha\left(k_{1} \wedge \ell_{1}\right)=\beta\left(f^{\prime}\left(k_{1}\right) \wedge g^{\prime}\left(\ell_{1}\right)\right)=\beta\left(f^{\prime}\left(k_{2}\right) \wedge g^{\prime}\left(\ell_{1}\right)\right)=\alpha\left(k_{2} \wedge \ell_{1}\right)=1,
$$

which is a contradiction. Also, since $\beta\left(f^{\prime}\left(k_{2}\right) \wedge g^{\prime}\left(\ell_{1}\right)\right)=\alpha\left(k_{2} \wedge \ell_{1}\right)=1$, it follows that $f^{\prime}\left(k_{2}\right)$ is not the basepoint of $K^{\prime}$. Since $\beta\left(f^{\prime}\left(k_{1}\right) \wedge g^{\prime}\left(\ell_{2}\right)\right)=\alpha\left(k_{1} \wedge \ell_{2}\right)=1$, also $f^{\prime}\left(k_{1}\right)$ is not the basepoint. Thus the two non-basepoints $f^{\prime}\left(k_{1}\right) \neq f^{\prime}\left(k_{2}\right)$ in $K^{\prime}$ are distinct. Whence, there are no elements $x_{0} \wedge y_{0} \in \mathrm{SP}\left(S^{0}\right) \wedge \mathrm{SP}\left(S^{0}\right)$ such that identifications of type (3) occur with the element $\alpha \wedge x_{K} \wedge y_{L}$.

Therefore, we have presented a non-trivial element $\alpha \wedge x_{K} \wedge y_{L} \in \mathcal{W}\left(K \wedge L, S^{0}\right) \wedge$ $\mathrm{SP}\left(S^{0}\right) \wedge \mathrm{SP}\left(S^{0}\right)$ in the summand $(\mathrm{SP} \wedge \mathrm{SP})\left(S^{0}\right)$ of equation (3.4) that is not identified with any element in $\mathrm{SP}\left(S^{0}\right) \wedge \mathrm{SP}\left(S^{0}\right)$. Thus $(\mathrm{SP} \wedge \mathrm{SP})\left(S^{0}\right) \not \operatorname{SP}\left(S^{0}\right) \wedge \operatorname{SP}\left(S^{0}\right)$.

Remark 3.15. In Example 3.14, we purposefully have chosen a $\mathcal{W}$-space $X$ where the structure maps:

$$
K \wedge X\left(S^{0}\right) \rightarrow X\left(K \wedge S^{0}\right) \cong X(K)
$$

are not epimorphisms. However, if $X$ is an $\mathbb{S}$-coalgebra in $\mathcal{W}$-spaces, Lemma 3.6 shows that such non-epimorphic structure maps are not possible.

Example 3.16. Recall, from [MMSS01, Example 4.8], that if we choose $\mathcal{D}$ to be the category of finite based sets $n_{+}=\{0,1, \ldots, n\}$ and all based maps, where 0 is the basepoint, then a $\mathcal{D}$-space is a $\Gamma$-space as in [Seg74]. As in $\mathcal{W}$-spaces, here the sphere spectrum $\mathbb{S}: \mathcal{D} \rightarrow$ Top $_{*}$ is defined as the strong symmetric monoidal faithful functor induced by inclusion (endowing the finite sets with the discrete topology). 
Recall from [MMSS01, Lemma 4.9] that we have $X \wedge_{\mathbb{S}} Y \cong X \wedge Y$ for any $\Gamma$-spaces $X$ and $Y$, as the action of the sphere spectrum $\mathbb{S}$ provides no additional data. As in Example 3.14, the $\Gamma$-space model of the Eilenberg-Mac Lane spectrum $H \mathbb{Z}$ provides an example of a $\Gamma$-space $X$ where $(X \wedge X)\left(1_{+}\right) \not X\left(1_{+}\right) \wedge X\left(1_{+}\right)$. The proof is similar to Example 3.14.

\section{Coalgebras in EKMM-spectra}

We prove here (in Theorem 4.1 below) a result similar to Theorem 3.4 for EKMMspectra using the same strategy from Theorem 2.2. We first investigate the definition of the smash product in this case.

Let us set notation and recall the definitions. All missing details can be found in [EKMM97]. Let $\mathcal{L}$ denote the category whose objects are universes and whose morphisms are linear isometries. We fix $\mathcal{U}$ a universe, that is, a countable dimensional real inner product space. We say $X$ is a spectrum indexed on $\mathcal{U}$ if we are given a collection $X_{V}$ of pointed topological spaces for each $V \subseteq \mathcal{U}$ a finite dimensional subspace, together with structure maps:

$$
\sigma_{V, W}: \Sigma^{W-V} X_{V} \longrightarrow X_{W}
$$

whenever $V \subseteq W \subseteq \mathcal{U}$, such that the adjoint of each $\sigma_{V, W}$ is a homeomorphism. Given two spectra $X$ and $Y$ indexed on $\mathcal{U}$, their external smash product $X \bar{\wedge} Y$ is a spectrum indexed on $\mathcal{U}^{\oplus 2}$ defined by:

$$
(X \bar{\wedge} Y)_{V}=X_{V_{1}} \wedge Y_{V_{2}}
$$

for any finite dimensional subspace $V=V_{1} \oplus V_{2} \subseteq \mathcal{U}^{\oplus 2}$. Let $\mathcal{L}(n)=\mathcal{L}\left(\mathcal{U}^{\oplus^{n}}, \mathcal{U}\right)$ for all $n \in \mathbb{N}$. We say $X$ is an $\mathbb{L}$-spectrum if the spectrum $X$ is endowed with an action $\alpha: \mathcal{L}(1) \ltimes X \longrightarrow X$ (see [EKMM97, Chapter I, Definition 4.2]). If $X$ and $Y$ are $\mathbb{L}$-spectra, their operadic smash product $X \wedge_{\mathcal{L}} Y$ is the coequalizer:

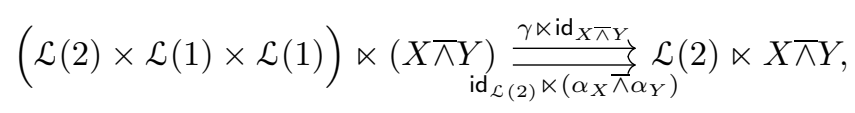

where $\gamma: \mathcal{L}(2) \times \mathcal{L}(1) \times \mathcal{L}(1) \rightarrow \mathcal{L}(2)$ is the map defined by: $(\theta, \varphi, \psi) \longmapsto \theta \circ(\varphi \oplus \psi)$, and $\operatorname{id}_{\mathcal{L}(2)} \ltimes\left(\alpha_{X} \bar{\wedge} \alpha_{Y}\right)$ is defined via the isomorphism (see [EKMM97, Chapter I, Proposition 2.2(ii)]):

$$
(\mathcal{L}(2) \times \mathcal{L}(1) \times \mathcal{L}(1)) \ltimes X \bar{\wedge} Y \cong \mathcal{L}(2) \ltimes((\mathcal{L}(1) \times \mathcal{L}(1)) \ltimes(X \bar{\wedge} Y)),
$$

and $\alpha_{X} \bar{\wedge} \alpha_{Y}$ is the induced map:

$$
\mathcal{L}(1) \times \mathcal{L}(1) \ltimes(X \bar{\wedge} Y) \cong(\mathcal{L}(1) \ltimes X) \bar{\wedge}(\mathcal{L}(1) \ltimes Y) \stackrel{\alpha_{X} \bar{\wedge} \alpha_{Y}}{\longrightarrow} X \bar{\wedge} Y .
$$

A definition of the twisted half-smash product $\mathcal{L}(2) \ltimes X \bar{\wedge} Y$ can be found in [Col97, Definition 5.1]. Let us make the construction explicit. For each $V \subseteq \mathcal{U}^{\oplus 2}$, define a Thom spectrum $\mathcal{M}(V)$ indexed on $\mathcal{U}$ so that, for any $V \subseteq W \subseteq \mathcal{U}^{\oplus 2}$ we have an isomorphism of spectra indexed on $\mathcal{U}$ :

$$
\Sigma^{W-V} \mathcal{M}(W) \stackrel{\cong}{\longrightarrow}(V),
$$

see [Col97, Proposition 4.3]. Whenever the dimension of $U \subseteq \mathcal{U}$ is strictly smaller 
than the dimension of $V \subseteq \mathcal{U}^{\oplus 2}$, the space $\mathcal{M}(V)_{U}$ is just a point (see beginning of [Col97, Section 4]). The twisted half-smash product $\mathcal{L}(2) \ltimes(X \bar{\wedge} Y)$ is defined to be the colimit (see [Col97, Definition 3.5]) in the category of spectra indexed over $\mathcal{U}$ :

$$
\operatorname{colim}_{V \subseteq \mathcal{U} \oplus 2}\left(\mathcal{M}(V) \wedge(X \bar{\wedge} Y)_{V}\right)
$$

where the colimit is taken over the maps:

$$
\begin{aligned}
\mathcal{M}(V) \wedge(X \wedge Y)_{V} & \cong \Sigma^{W-V} \mathcal{M}(W) \wedge\left(X \bar{\wedge}(W)_{V}\right. \\
& \cong \mathcal{M}(W) \wedge \Sigma^{W-V}(X \wedge Y)_{V} \\
& \rightarrow \mathcal{M}(W) \wedge(X \wedge Y)_{W}
\end{aligned}
$$

for $V \subseteq W \subseteq \mathcal{U}^{\oplus 2}$, finite dimensional subspaces. This amounts to saying that, for any finite dimensional subspace $U \subseteq \mathcal{U}$, the smash product $\left(X \wedge_{\mathcal{L}} Y\right)_{U}$ can be regarded as a quotient of the following space:

$$
\left(\bigvee_{\substack{V=V_{1} \oplus V_{2} \subseteq \mathcal{U}^{\oplus 2} \\ \text { finite dimensional }}} \mathcal{M}(V)_{U} \wedge X_{V_{1}} \wedge Y_{V_{2}}\right) / \sim .
$$

Since $\mathcal{M}(V)_{U}$ is a point whenever the dimension of $V$ is bigger than $U$ and that $\mathcal{M}(0)_{0} \cong S^{0}$, we obtain:

$$
\left(X \wedge_{\mathcal{L}} Y\right)_{0} \cong X_{0} \wedge Y_{0}
$$

Recall from [EKMM97, Chapter III, Definition 1.1] that an $\mathbb{S}$-module $X$ is an $\mathbb{L}$-spectrum such that the natural map $\mathbb{S} \wedge_{\mathcal{L}} X \rightarrow X$ is an isomorphism. The smash product of $\mathbb{S}$-modules $X$ and $Y$ is defined as $X \wedge_{\mathbb{S}} Y=X \wedge_{\mathcal{L}} Y$ (see [EKMM97, Chapter II, Definition 1.1]). We denote the resulting symmetric monoidal category by $\mathrm{Sp}$ EKMM . Similarly as in previous sections, given a commutative monoid $R$ in $\mathrm{Sp}^{\text {EKMM }}$ (i.e. a commutative $\mathbb{S}$-algebra), we define the smash product $X \wedge_{R} Y$ of two $R$-modules $X$ and $Y$ as the coequalizer:

$$
X \wedge_{\mathbb{S}} R \wedge_{\mathbb{S}} Y \underset{(\sigma \circ \tau) \wedge \mathrm{id}_{Y}}{\stackrel{\mathrm{id}_{X} \wedge \sigma}{\longrightarrow}} X \wedge_{\mathbb{S}} Y .
$$

See [EKMM97, Chapter III, Definition 3.1].

Theorem 4.1. Let $R$ be a commutative $\mathbb{S}$-algebra in $\mathrm{Sp}^{\mathrm{EKMM}}$ such that $R_{0} \cong S^{0}$. Let $(C, \Delta, \varepsilon)$ be an $R$-coalgebra in $\mathrm{Sp}{ }^{\mathrm{EKMM}}$. Then $C$ is cocommutative.

Proof. We apply Theorem 2.2 to the pair $R \wedge-: \operatorname{Top}_{*} \rightleftarrows \operatorname{Mod}_{R}\left(\mathrm{Sp}^{\mathrm{EKMM}}\right): \mathrm{Ev}_{0}$. Equation (4.3) shows that $\mathrm{Ev}_{0}$ is a strong symmetric monoidal functor, and thus proves condition (i). Lemma 2.4 shows (ii). We conclude using Lemma 4.2 below and (iii) of Theorem 2.2.

Lemma 4.2. Let $R$ be a commutative $\mathbb{S}$-algebra in $\mathrm{Sp}^{\mathrm{EKMM}}$. Let $(C, \Delta, \varepsilon)$ be an $R$ coalgebra in $\mathrm{Sp}$ EKMM . Then the natural map $\sigma: R \wedge C_{0} \longrightarrow C$ is an epimorphism of $R$-modules in $\mathrm{Sp} \mathrm{EKMM}^{\mathrm{T}}$.

Proof. As we have recalled above, given any finite dimensional subspace $U \subseteq \mathcal{U}$, the 
smash product $\left(C \wedge_{R} C\right)_{U}$ can be regarded as:

$$
\left(C \wedge_{R} C\right)_{U}=\left(\bigvee_{\substack{V=V_{1} \oplus V_{2} \subseteq \mathcal{U}^{\oplus 2} \\ \text { finite dimensional }}} \mathcal{M}(V)_{U} \wedge C_{V_{1}} \wedge C_{V_{2}}\right) / \sim_{R} .
$$

If $\operatorname{dim}(U)=1$ in equation (4.5), we get that $\left(C \wedge_{R} C\right)_{U}$ is simply:

$$
\left(\left(\mathcal{M}(U \oplus 0)_{U} \wedge C_{U} \wedge C_{0}\right) \vee\left(\mathcal{M}(0)_{U} \wedge C_{0} \wedge C_{0}\right) \vee\left(\mathcal{M}(0 \oplus U)_{U} \wedge C_{0} \wedge C_{U}\right)\right) / \sim_{R}
$$

Denote the action from the $\mathbb{L}$-structure of $C$ by $\alpha: \mathcal{L}(1) \ltimes C \longrightarrow C$. Elements in $C_{U} \wedge C_{0}$ are not identified with elements in $C_{0} \wedge C_{U}$ in the coequalizer (4.1) via the map $\alpha \bar{\wedge} \alpha$. However, elements of $C_{U} \wedge C_{0}$ can be identified with elements of $C_{0} \wedge C_{U}$ under the $R$-action $R_{U} \wedge C_{0} \rightarrow C_{U}$ (in the coequalizer (4.4)) and the structure map $\sigma_{0, U}$ in $\left(C \wedge_{R} C\right)_{U}$ (in the colimit (4.2)). Notice the similarity with equation (3.2) in previous section. We can argue similarly. Suppose the map $R_{U} \wedge C_{0} \rightarrow C_{U}$ is not surjective. Since the composite:

$$
S^{U} \wedge C_{0} \longrightarrow R_{U} \wedge C_{0} \longrightarrow C_{U}
$$

is the structure map $\sigma_{0, U}$, then $\sigma_{0, U}$ must also not be surjective. Let $c$ be an element in $C_{U}$ not in the image of $R_{U} \wedge C_{0} \rightarrow C_{U}$. Notice that $c$ is also not in the image of $\sigma_{0, U}$. Its preimage under the map $\varepsilon \wedge \mathrm{id}_{C}$ lies in $C_{0} \wedge C_{U}$ but its preimage under the map $\operatorname{id}_{C} \wedge \varepsilon$ lies in $C_{U} \wedge C_{0}$. Since $c$ is not in the image of $\sigma_{0, U}$, elements in the two preimages $\left(\operatorname{id}_{C} \wedge \varepsilon\right)^{-1}(c)$ and $\left(\varepsilon \wedge \mathrm{id}_{C}\right)^{-1}(c)$ are not identified in the colimit (4.2). Similarly, since $c$ is not in the image of $R_{U} \wedge C_{0} \rightarrow C_{U}$, the elements in the two preimages are not identified in the coequalizer (4.4). Thus, the two preimages $\left(\operatorname{id}_{C} \wedge \varepsilon\right)^{-1}(c)$ and $\left(\varepsilon \wedge \mathrm{id}_{C}\right)^{-1}(c)$ are disjoint in $\left(C \wedge_{R} C\right)_{U}$. But we then get a contradiction with the commutativity of the diagram:

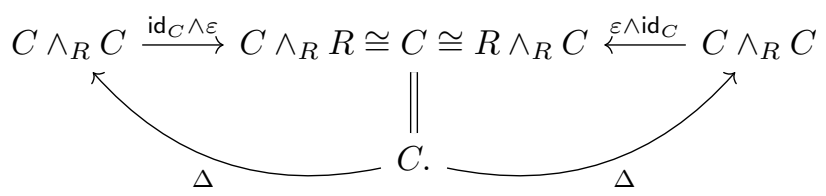

The higher dimensional cases are done similarly, so the map $R \wedge C_{0} \longrightarrow C$ is an epimorphism.

\section{References}

[AGP02] M. Aguilar, S. Gitler, and C. Prieto, Algebraic Topology from a Homotopical Viewpoint, Universitext, Springer-Verlag, New York, 2002, Translated from the Spanish by S.B. Sontz. MR 1908260.

[AM10] M. Aguiar and S. Mahajan, Monoidal Functors, Species and Hopf Algebras, CRM Monogr. Ser., vol. 29, American Mathematical Society, Providence, RI, 2010, with forewords by K. Brown and S. Chase and A. Joyal. MR 2724388. 
[And74] D.W. Anderson, Convergent functors and spectra, Localization in Group Theory and Homotopy Theory, and Related Topics (Sympos., Battelle Seattle Res. Center, Seattle, Wash., 1974), Lecture Notes in Math., vol. 418, Springer, Berlin, 1974, pp. 1-5. MR 0383388.

[BF78] A.K. Bousfield and E.M. Friedlander, Homotopy theory of $\Gamma$-spaces, spectra, and bisimplicial sets, Geometric Applications of Homotopy Theory (Proc. Conf., Evanston, Ill., 1977), II, Lecture Notes in Math., vol. 658, Springer, Berlin, 1978, pp. 80-130. MR 513569.

[Col97] M. Cole, Appendix A: Twisted half-smash product and function spectra, Rings, Modules, and Algebras in Stable Homotopy Theory, Math. Surveys Monogr., vol. 47, American Mathematical Society, 1997.

[EKMM97] A. Elmendorf, I. Kriz, M.A. Mandell, and J.P. May, Rings, Modules, and Algebras in Stable Homotopy Theory, Math. Surveys Monogr., vol. 47, American Mathematical Society, Providence, RI, 1997, with an appendix by M. Cole. MR 1417719.

[HS] K. Hess and B. Shipley, Topological coHochschild homology (coTHH), in preparation.

[HSS00] M. Hovey, B. Shipley, and J. Smith, Symmetric spectra, J. Amer. Math. Soc. 13 (2000), no. 1, 149-208. MR 1695653.

[Lur18] J. Lurie, Elliptic cohomology I: Spectral abelian varieties, http:// www.math.harvard.edu/ lurie/papers/Elliptic-I.pdf, February 2018, preprint.

[MM02] M.A. Mandell and J.P. May, Equivariant orthogonal spectra and S-modules, Mem. Amer. Math. Soc. 159 (2002), no. 755, x+108. MR 1922205.

[MMSS01] M.A. Mandell, J.P. May, S. Schwede, and B. Shipley, Model categories of diagram spectra, Proc. Lond. Math. Soc. (3) 82 (2001), no. 2, 441512. MR 1806878.

[Sch] S. Schwede, Symmetric Spectra, http://www.math.uni-bonn.de/ people/schwede/SymSpec-v3.pdf, electronic book.

[Seg74] G. Segal, Categories and cohomology theories, Topology 13 (1974), 293-312. MR 0353298.

[Shi04] B. Shipley, A convenient model category for commutative ring spectra, Homotopy Theory: Relations with Algebraic Geometry, Group Cohomology, and Algebraic K-Theory, Contemp. Math., vol. 346, American Mathematical Society, Providence, RI, 2004, pp. 473-483. MR 2066511.

Maximilien Péroux mholmb2@uic.edu

Brooke Shipley shipleyb@uic.edu

Department of Mathematics, Statistics and Computer Science, University of Illinois at Chicago, 851 S. Morgan Street, Chicago, IL, 60607-7045, USA 